\title{
Ergonomics Importance of Wearable Medical Devices
}

\author{
Aksahin $\mathrm{MF}^{*}$ \\ Biomedical Engineering Department, Baskent University, Turkey
}

*Corresponding author: Mehmet Feyzi Aksahin, Biomedical Engineering

Department, Baskent University, Turkey, Email: maksahin@baskent.edu.tr

\section{Mini Review \\ Volume 1 Issue 3}

Received Date: November 13, 2017

Published Date: November 22, 2017

DOI: $10.23880 /$ eoij-16000124

\section{Abstract}

Medical technologies directly related to human and developed for diagnosis and/or treatment of diseases, are one of the most important issues of today. In order to prevent the various problems that arise with the increase of the human population, researchers are trying to give mobility to these technologies. Wearable medical devices are being developed for this purpose. In this study, the ergonomic importance of wearable medical devices in the literature is briefly summarized.

Keywords: Wearable Medical Device; Sensor Interaction; Thermal Aspects; Aesthetics; Human Movement; Proxemics

\section{Introduction}

Medical technology has shown great progress in diagnosis and treatment of diseases from past to present. As a result of this progress in device developing, treatment and diagnosis of many diseases can be made more effectively and with high accuracy. By the developments on medical technology, solutions can be produced fortemporal and spatial problems arising from the growing human population. These problems can only be overcome by the uninterrupted and continuous supply of medical interaction between patients. At this point, it is very important to bring mobility to medical devices. Evolving medical technology has made the devices wearable instead of the patients moving alongside the devices.Wearable medical devices have more complex instrumentation. In fact, complex structures are designed with the opportunities provided by the developing technology and give accurate results. In addition to measuring high-precision results, it is important that the patient can easily use the device. In order to provide this, orienting the ergonomic device design beside the engineering principles will provide convenience for both the patient and the physician.

A wearable medical device can be defined as a device that is autonomous, noninvasive, and that performs a specific medical function such as monitoring or support over a prolonged period of time [1]. In literature; there are many studies about ergonomics of wearable medical devices. Ergonomics and wearability deal with a wide range of design issues including the physical shape of wearable, their active relationship with the human anatomy in motion, their acceptability as a function of comfort, fashion, and purpose, the relationship between the wearable device and the work environment, the physical factors that affect their use, and the humandevice interaction [2]. Physical design which is one of the most important point on many studies in literature. It refers to issues dealing with thephysical shape, size, and weight of a wearable and its ergonomics [1]. For the efficient wearable medical device design can be done by applying general design guidelines during the designing stage. These guidelines can be listed as placement, form language, human movement, proxemics, sizing, attachment, containment, weight, accessibility, sensor interaction, thermal aspects, aesthetics and long-term effect [3].

In a study of Axisa, et al. [4], it is developed smart and flexible sensors for healthcare and illness prevention. As clothes and textiles are in direct contact with about $90 \%$ of the skin surface, smart sensors and smart clothes with noninvasive sensors are an attractive solution for home-based and ambulatory health monitoring [4]. They studied on ambulatory device for use on the wrist. Also, it is mentioned that wrist is the 


\section{Ergonomics International Journal}

most convenient place to wear an ambulatory device because the attachment of device is easier on wrist. Moreover, for the mobility, the wrist enables good ergonomics. Addition to this ambulatory device, there are three different wearable medical device are produced which are Modular Autonomous Recorder System for Measurement of ANS (MARSIAN) [5], Advanced Care Alert Portable Telemedical Monitor (AMON) [6] and Vivago Wrist Care [7].

Wee [8] mentions that medical home monitoring device systems portability for elderly. It is asked to old people that what the features of these devices are for motivate them. It is explained by old people that they are avoid of showing their sickness to other people. So, in their opinion wearable medical devices should be hidden.

Torres, et al. [9] describes a physical monitoring system that uses remarkable technology to assess exercise performance and patient progress. Developed devices are designed through the general designing guidelines for wearability to evaluate fourteen wearable device ergonomics. The evaluation shows that it is identified as useful elements that provide feedback to the user by therapists.

Raynolds [10] mentions about a specific wearable medical device. The Selectively Heated Therapeutic Sweater allows the patient freedom to choose where and when heat treatment for rheumatic diseases is applied to their joints throughout the day. It also takes into consideration their right to privacy and makes the treatment as unobtrusive to daily life as possible. Varadana, et al. [11] informs about textile basedmultielectrode system that can detect same EEG waves. Furthermore, this type of textile based system is ergonomic and can enable early diagnosis of Autism Spectrum Disorder (ASD) on infants.

Lymberis, et al. [12] explains the intelligent biomedical clothing to monitor body kinematics, analyze posture and gestures with major application for ergonomic, virtual reality, rehabilitation and treatment follow-up. Lmberis, et al. [13] mentions that in smart wearable health systems and applications, non-invasive sensors are particularly suitable for humans. These sensors are high complex and have difficulties on measuring deep phenomenon from the surface skin. These phenomenon's relate to the localizations are limited with respect to the ergonomics.

Page [14] informs the reasons for why people do not choose wearable technology. To change their idea, this type of devices should be more tested with end users. Also, the products should be more ergonomic.
Ehmen, et al. [15] compares usability and acceptability of four different wearable ECG or heart rate measuring devices. They conclude that the creation of an acceptable monitoring device for older people. This device requires designers and developers to consider their special demands and abilities with device ergonomic.

\section{Discussion and Conclusion}

It can be seen that there are not too much publications about wearable medical devices in literature. It is also understoodfrom existing articles that wearable medical devices and ergonomics are directly related to each other. Designers who develop wearable medical devices should be focused on ergonomics to prevent from dissatisfactions of patients. By this, wearable devices will be more acceptable. By increase in articles about ergonomics of wearable medical devices will largely close the gap in the literature.

\section{References}

1. Fotiadis DI, Glaros C, Likas A (2006) Wearable medical devices. Wiley Encyclopedia of Biomedical Engineering.

2. Baber C, Knight J, Haniff D, Cooper L (1999) Ergonomics of wearable computers. Mobile Networks and Applications 4(1): 15-21.

3. Goodwin MS, Velicer WF, Intille SS (2008) Telemetric monitoring in the behavior sciences. Behavior research methods 40(1): 328341.

4. Axisa F, Schmitt PM, Gehin C, Delhomme G, McAdams E, et al. (2005) Flexible technologies and smart clothing for citizen medicine, home healthcare, and disease prevention. IEEE Transactions on information technology in biomedicine 9(3): 325-336.

5. Axisa F, Dittmar A, Delhomme G (2003) Smart clothes for the monitoring in real time and conditions of physiological, emotional and sensorial reactions of human. In Engineering in Medicine and Biology Society, Proceedings of the 25th Annual International Conference of the IEEE 4: 3744-3747.

6. AMON Project.

7. VIVAGO System.

8. Wee WTB (2008) A mini-study of Singapore elderly acceptance to portable medical home systems. In Proceedings of the 2nd International Convention 


\section{Ergonomics International Journal}

on Rehabilitation Engineering \& Assistive Technology, Singapore Therapeutic, Assistive \& Rehabilitative Technologies (START) Centre, pp: 271-276.

9. Torres A, López G, Guerrero LA (2016) Using nontraditional interfaces to support physical therapy for knee strengthening. Journal of medical systems 40(9): 194.

10. Reynolds AH (2010) Resistively heated fabrics for use in wearable therapeutic devices by Aria $\mathrm{H}$. Reynolds (Doctoral dissertation, Massachusetts Institute of Technology).

11. Varadana VK, Raib P, Ohb SC, Kumar bPS (2016) Wearable Technology and Mobile Platform for Human Health Monitoring. FERMAT 16: 1-38.

12. Lymberis $A$, Olsson $S$ (2003) Intelligent biomedical clothing for personal health and disease management: state of the art and future vision. Telemedicine Journal and e-health 9(4): 379-386.

13. Lmberis A, Dittmar A (2007) Advanced wearable health systems and applications-research and development efforts in the European Union. IEEE Engineering in Medicine and Biology Magazine 26(3): 29-33.

14. Page $\mathrm{T}$ (2015) A forecast of the adoption of wearable technology. International Journal of Technology Diffusion 6(2): 12-29.

15. Ehmen H, Haesner M, Steinke I, Dorn M, Gövercin $M$ et al. (2012) Comparison of four different mobile devices for measuring heart rate and ECG with respect to aspects of usability and acceptance by older people. Applied ergonomics 43(3): 582-587. 